\title{
Apport de l'échelle nanométrique en thérapie photodynamique,la lumière à l'assaut du cancer:quoi de neuf?
}

\author{
Contribution of nanometres scale in photodynamic therapy, light to fight \\ cancer:what's new?
}

\author{
Khadidja YANALAH 1-2, Nassima MOUSSAOUI 1-2 \\ 1 Laboratoire de pharmacie galénique \\ 2 Faculté de Médecine d'Oran,université Oran 1
}

Article: (Yahui Wu a,1, Fangnian Li) et al. «Tumor microenvironment-responsive PEGylated heparin-pyropheophorbide- a nano conjugates for photodynamic therapy. "Carbohydrate Polymers 2552021 (117490).https://doi.org/10.1016/j. carbpol.2020.117490

Citation: YANALAH, K.et al. Apport de l'échelle nanométrique en thérapie photodynamique, la lumière à l'assaut du cancer : quoi de neuf ? (2021) J Fac Med Or 5(1):679-680

DOI : https://doi.org/ 10.51782/jfmo.v5i1.121
Le développement d'un médicament efficace contre le cancer est l'un des plus grands défis de santé auxquels l'humanité est confrontée. Les effets secondaires indésirables de la chimiothérapie dans le traitement du cancer ont incité les scientifiques à rechercher des traitements innovants et moins funestes.La photothérapie dynamique s'est imposée comme la meilleure alternative pour s'éloigner des effets secondaires indésirables de la chimiothérapie.Cependant, la biomédecine s'oriente vers l'utilisation denanoparticules photosensibilisantes pour réduire les effets indésirables de cette technique.

Cet article rappelle brièvement le principe de la thérapie photodynamique puis présente les dernières avancées en termes d'applications cliniques.

\section{Introduction}

La Thérapie Photodynamique (PDT) est une photochimiothérapie basée sur l'activation par la lumière de photosensibilisants non toxiques à l'obscurité. Après excitation lumineuse (lumière laser en général) et en présence d'oxygène, les photosensibilisants génèrent des espèces réactives de l'oxygène dont l'oxygène singulet, qui entraine des réactions de photo-oxydation induisant ainsi, des altérations irréversibles des lipides membranaires, des protéines ou des acides nucléiques des cellules, ayant pour conséquence, la mort cellulaire par nécrose et/ou apoptose [1].

Les photosensibilisants habituellement utilisés sont des molécules fortement hydrophobes qui s'agrègent en milieux aqueux biologique, limitant ainsi leur biodisponibilité. De ce fait, utilisées seules, ces molécules nécessitent d'être injectées à des concentrations élevées, engendrant un risque de photosensibilisation générale.

Pour diminuer cet effet secondaire et rendre le traitement plus efficace, certains auteurs ont orienté leurs recherches vers l'encapsulation des photosensibilisants, développant ainsi des nanovecteurs, notamment des nanoparticules capables de reconnaitre spécifiquement et indirectement les cellules cancéreuses [2].

L'intérêt, en est de limiter les effets non spécifiques des photosensibilisants tout en assurant une concentration élevée au niveau même de la tumeur. 


\section{Des stratégies prometteuses pour la nanovectori- sation des photosensibilisants}

La nanomédecine par l'utilisation de nanoparticules permettrait d'améliorer le ciblage tumoral car ces dernières sont capables de s'accumuler spontanément dans les tumeurs solides grâce à l'effet de perméabilité et de rétention accrue [3].

Par ailleurs, l'héparine, un polysaccharide naturel, est considérée comme un vecteur prometteur d'administration de médicaments antitumoraux en raison de ses excellentes propriétés, telles qu'une grande biocompatibilité, biodégradabilité et l'inhibition de l'angiogenèse [4].

En outre, l'héparine se lie avec une grande affinité aux cellules endothéliales vasculaires en division et attire une attention intense pour améliorer l'efficacité du ciblage des tumeurs solides pour la thérapie tumorale, car les tissus tumoraux sont riches en cellules endothéliales.

Ces propriétés font de l'héparine un candidat prometteur pour la nanovectorisation afin de délivrer des photosensibilisants dans les cellules tumorales pour la thérapie photodynamique.

\section{Quels résultats pour la nanovectorisation des pho- tosensibilisants?}

Des résultats intéressants ont pu être obtenus à l'issue d'une étude réalisée chez des souris de souche «BALB/C » porteuses de la tumeur 4T1; les animaux ont reçu une injection intraveineuse de nanoparticules à base d'héparine PEGylée (HP). Un laser de $660 \mathrm{~nm}$ a été utilisé pour irradier les tumeurs 48 heures après l'injection.

L'absorption cellulaire de nanoparticules d'héparine par les cellules 4T1 a conduit à une production abondante d'espèces réactives de l'oxygène après irradiation, induisant l'apoptose cellulaire.

En effet, l'héparine PEGylée (HP) agrégée en nanoparticules, a permis d'atteindre la plus grande accumulation tumorale, la durée de rétention la plus longue et la meilleure pénétration dans les tissus tumoraux, ce qui a entraîné la plus grande efficacité anticancéreuse in vivo avec un taux d'inhibition de la croissance tumorale de $94,3 \%$. Suggérant que les nanomédicaments à base de HP PEGylée sensibles au microenvironnement tumoral peuvent agir comme des agents anticancéreux efficients.

\section{Conclusion}

Il ressort que ce type de nanoplatforme peut être étendu pour concevoir des nanomédicaments efficaces et biocompatibles pour la thérapie photodynamique à partir d'autres photosensibilisateurs ou agents anticancéreux pour la thérapie du cancer.

\section{Références bibliographiques}

[1] Dougherty, Thomas J., et al. «Photodynamic therapy.» JNCl: Journal of the national cancer institute 90.12 (1998): 889-905.

[2] Maxime DEMAZEAU, Relations structure-effet de nanovecteurs à base de copolymères à blocs pour la thérapie photodynamique : Utilisation de modèles de membranesthèseen vue de l'obtention dudoctorat de l'université de toulouse,le 4 octobre 2019

[3] Ludovic Bretin,Thérapie photodynamique (PDT) dans un modèle in vitro et in vivo de cancer colorectal : utilisation d'un photosensibilisateur nanovectorisé,https://tel.archives-ouvertes.fr/tel-0244321

[4] Newland, B., Varricchio, C., Korner, Y., Hoppe, F., Taplan, C., Newland, H., et al. (2020). Focal drug administration via heparin-containing cryogelmicrocarriers reduces cancer growth and metastasis. Carbohydrate Polymers, 245, Article 116504. 